\title{
A cyclic progressive collapse method to predict the bending response of a ship hull girder
}

\author{
S. Li, Z.Q. Hu \& S.D. Benson \\ Marine, Offshore and Subsea Technology Group, School of Engineering, Newcastle University, UK
}

\begin{abstract}
This paper describes a cyclic progressive collapse method for the prediction of ship hull girder cyclic bending response. The proposed methodology extends the established simplified progressive collapse method, which is only capable of simulating the monotonic bending moment-curvature relationship. However, the actual hull girder collapse can involve multiple load cycles, such as that caused by a series of storm waves. During these load cycles, the onset of plasticity and local buckling can reduce the overall strength and stiffness of the hull girder. Hence, a cyclic progressive collapse method is proposed to predict the ship hull girder structural response under cyclic bending. This method follows the major assumptions and procedure embedded in the original Smith method with an extended capability to re-formulate the load-shortening curve of structural element under cyclic loading. The proposed methodology is applied to predict the cyclic bending responses of two box girder models. Nonlinear finite element analysis is performed as a validation. The present study shows the feasibility of the proposed cyclic progressive collapse method, but also suggests the need to develop an enhanced model to predict the cyclic behaviour of structural components.
\end{abstract}

\section{INTRODUCTION}

The ultimate limit state approach, pioneered by Caldwell (1965), has become an established practical methodology to evaluate the ship hull girder bending strength and the simplified progressive collapse method (Smith method) introduced by Smith (1977) has been incorporated in classification society rules. Although it is a capable method in terms of the estimation of ultimate bending strength, as shown by the experimental validation of Dow (1991), the simplified progressive collapse method is limited to the prediction of monotonic bending response from pre-ultimate to postultimate regimes. However, the actual failure of a ship hull girder can involve multiple loading cycles, such as that caused by a series of storm waves.

In this regard, it is critical to predict the cyclic bending response of a ship hull girder. Therefore, this paper aims to extend the capability of the established simplified progressive collapse method to predict the cyclic bending response of a ship hull girder. The proposed methodology follows the major assumptions and procedure in the original approach. The extension is attributed to the reformulation of load-shortening curve of structural components. The overall algorithm is described and a case study is performed on two box girder models. Additionally, nonlinear finite element analysis is carried out as a validation.

\section{BACKGROUND}

Both 14th (2000) and 15th (2003) ISSC have discussed the cyclic failure of ship hull girders. It was indicated that the static monotonic response and the corresponding collapse strength may only be applicable to the single load excursion related to the incorrect operation of loading and unloading, such as the failure of Energy Concentration. The structural capacity under cyclic loading can be significantly lower than the instantaneous collapse strength under a single load excursion, since the reversal and accumulation of plastic deformation can permanently degrade the structural resistance against subsequent loading and consequently lead to an irreversible collapse. This is supported by the failure analysis of an inland waterway oil bunker barge performed by Hess et al. (1997). It was reported that the cause of the barge collapse was largely attributed to the progressive damage, which resulted in inelastic behaviour during repeated cycles of loading. As suggested by the study of Sumi et al. (2015) and also discussed in the 20th ISSC (2018), the accident of MOL Comfort was likely a dynamic cyclic process where the collapse progressed over more than one cycle of loading and was a factor on its eventual failure. This may further exemplify the importance of investigating the cyclic failure of ship girder. To this respect, Iijima and Fujikubo (2015) experimentally investigated 
the cumulative damage and the collapse extent of a simple scaled model under a series of extreme waves. Meanwhile, a hydro-elastoplasticity method is proposed to simulate the dynamic collapse of ship hull girders (Iijima et al., 2011). In this methodology, the nonlinear stiffness term is approximated either by a few piece-wise linear curves or a series of spline curves which smoothly interpolate the measured points in a static test. The unloading stiffness is taken as the same as the initial stiffness and the reloading response is assumed to follow the same path as the initial response (Figure 1). Although it was employed to predict the dynamic collapse under a series of focused waves, this method should limit itself to predict the response under a half periodic wave since the structural stiffness during reloading needs to be formulated in a different way. Derbanne et al. (2016) adopted a similar approach to study the dynamic ultimate strength under whipping for 14 container ships modelled as single-degree-of-freedom beams. The applied loading protocol predominantly oscillates in one direction. Therefore, a monotonic nonlinear stiffness term may be applicable as the overloading to the reversed direction was not required. However, a re-formulation must be carried out for the nonlinear stiffness term when a large reloading takes place.

Yao and Nikolov (1990) performed a series of finite element analysis on the unstiffened plates under in-plane cyclic tension and compression. Stem from the observation in this numerical study, Yao et al. (1995) later proposed an analytical method to predict the cyclic response of plates based on the elastic large deflection analysis and rigid-plastic mechanism analysis. Fukumoto and Kusama. (1985) experimentally and analytically studied the behaviour of a thin-walled beam under cyclic bending. An experimental

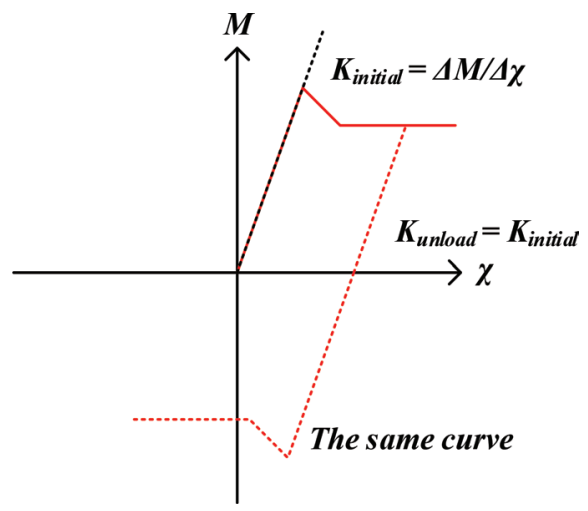

Figure 1. The nonlinear variation of structural stiffness assumed in the hydro-elastoplasticity method. programme was carried out by Kaminski (1992) to investigate the load-carrying capacity of a series of imperfect plates being cyclically compressed. Recently, an experimental test on a steel box girder was conducted by Cui and Yang (2018) to investigate the ultimate strength and failure characteristics under cyclic bending moment. Overall, the investigation of cyclic loading associated with ship structure ultimate strength are fairly limited in the literature compared to the monotonic loading. No theoretical methodology has been proposed to predict the cyclic bending response of a stiffened hull girder structure. Nonlinear finite element method can be used to perform such simulation but the drawback is also significant, such as the high computational cost and the uncertainty caused by the different finite element solvers, setups and parameters etc.

\section{METHODOLOGY}

\subsection{The simplified progressive collapse method}

The established simplified progressive collapse method predicts the ship hull girder's monotonic bending response through the following assumptions and procedure:

Assumptions:

1. Cross section remains plane during the progressive collapse;

2. No interaction exists between the adjacent structural elements;

3. The transverse frame is stocky enough such that the structural elements fail in an inter-frame mode.

Procedure:

1. The ship hull cross section is sub-divided into structural element;

2. Calculate the initial position of neutral axis;

3. A load-shortening curve (LSC) characterizing the response under in-plane load is assigned to each element;

4. Evaluate the tangent stiffness of each element at present strain using the load-shortening curve;

5. Evaluate the bending stiffness of the cross section with respect to the instantaneous neutral axis;

6. Apply the curvature increment about the instantaneous neutral axis;

7. Calculate the strain increment of each element

8. Evaluate the stress increment of each element using the load-shortening curve;

9. The bending moment increment is obtained either by the multiplication of bending stiffness and curvature increment or the integration of resultant stress of each element; 
10. Obtain the cumulative bending moment and curvature;

11. Update the position of instantaneous neutral axis;

12. Return to step 4.

\subsection{The cyclic progressive collapse method}

The proposed methodology continues to follow the assumptions and procedure of the simplified progressive collapse method described above, but re-formulates the LSC to account for the cyclic loading effect. The overall algorithm can be described with reference to Figure 2. The re-formulation of LSC is driven by a loading protocol which allows the direction of each curvature increment to be controlled. When the direction of curvature increment is reversed, the re-formulation of LSC of each element will be activated. This updated LSC will be utilized for the subsequent Smith method calculation with standard procedure until the next curvature increment reversal.

\subsection{LSC re-formulation scheme}

The LSC re-formulation scheme consists of a response rule and an updating rule. It is derived based the observation predicted by finite element analysis on the behaviour of unstiffened plate under cyclic in-plane compression/tension.

\subsubsection{Finite element analysis}

In light of investigating the load-shortening characteristics of structural components under cyclic

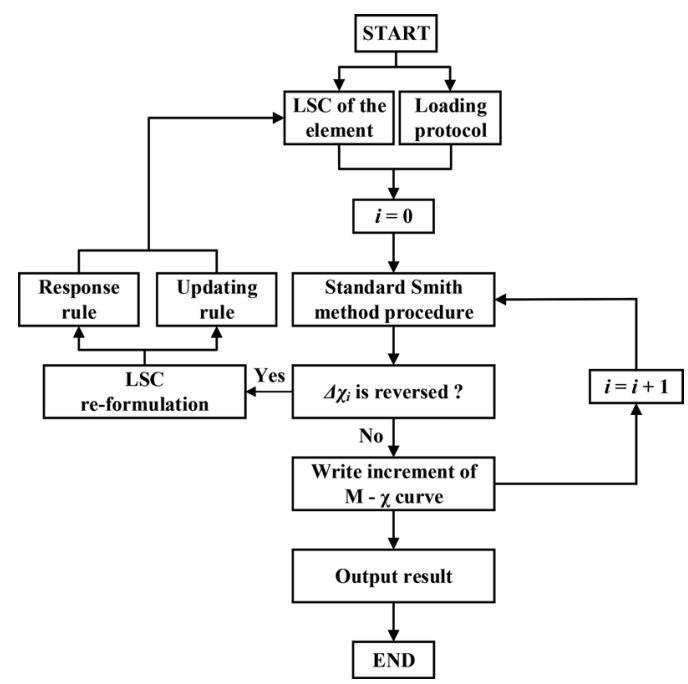

Figure 2. The overall algorithm of the proposed cyclic progressive collapse method. loading, a parametric finite element analysis is performed on a series of unstiffened plates. This parametric investigation covers four different aspect ratios $(\mathrm{a} / \mathrm{b}=1 \sim 4)$ and three different slenderness ratios $(\beta=1.5 \sim 2.5)$.

The applied loading protocol varies from singlecycle loading to multi-cycle loading, while the loading amplitude includes constant amplitude and varied amplitude. The constant amplitude implies that a same load (in terms of strain) is applied in each loading direction at each cycle, whereas a different load is applied in the varied amplitude loading.

A dynamic explicit solver is used for all analyses, which enables the application of the cyclic loading protocol. A complete cycle is set at $64 \mathrm{sec}-$ onds to ensure the quasi-static response and this is checked with equivalent static solution using arc-length method. The mesh size and boundary condition are consistent with the established practice for nonlinear analysis of plate and stiffened panel.

Typical cyclic load-shortening curves obtained by the finite element analysis are shown in Figure 3. It is evident that if the plate is loaded beyond the failure point under compression, the reloading from compression to tension experiences a highly nonlinear response with a stiffness much lower than the material Young's modulus. If no tension has been previously applied, it appears that the failure point in tension is close to the failure point under a monotonic tensile loading $\left(\varepsilon_{u} / \varepsilon_{y}=-1\right)$, as indicated by the dash line showing the monotonic tensile curve in Figure 3(a). However, if a tensile unloading has taken place previously, the tensile response path tends to approach the tensile unloading point (Figure $3 \mathrm{c}$ ).

There is a compressive ultimate strength reduction as shown in Figures 3(a) and 3(b). The reduction under varied amplitude loading is much larger than that under constant amplitude loading. This difference may relate to the fact that an equally large tension is applied in the constant amplitude case, whereas the tension loading is only applied up to the failure point in the varied amplitude case.

The foregoing general description applies to most of the unstiffened plates with various configurations in the parametric finite element analysis.

\subsubsection{Response rule}

In the present study, the LSC is idealized as piecewise linear and is formulated by several critical points, including:

- Permanent deformation point, at which the resultant stress is zero;

- Compressive/tensile unloading point, from which the unloading is started; 


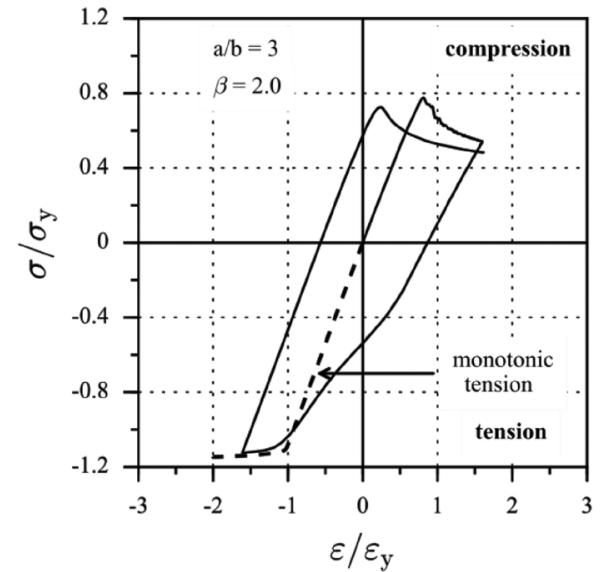

(a) Constant amplitude (Initial load: compression)

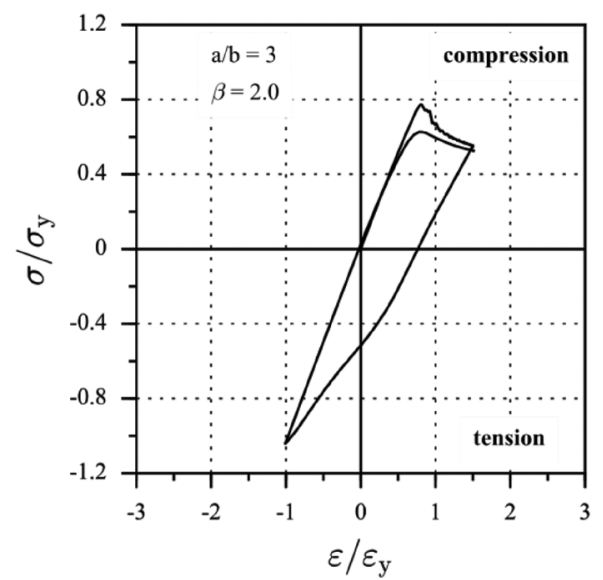

(b) Varied amplitude (Initial load: compression)

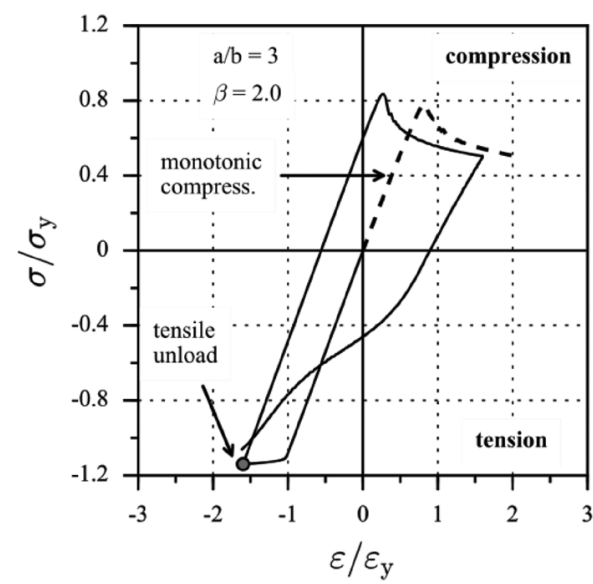

(c) Constant amplitude (Initial load: tension)

Figure 3. LSC predicted by finite element analysis of unstiffened plate under three different loading protocols.
- Compressive ultimate point. Paik's empirical formula (1997) is used to estimate the compressive ultimate strength;

- Tensile ultimate point. The tensile ultimate strength is assumed as the material yield stress;

- In both compression and tension, the ultimate strain is taken as the material yield strain.

Under compression, the pre-ultimate regime of the compressive LSC follows the path 'permanent deformation point $\rightarrow$ compressive ultimate point'. In the post-ultimate regime, the post-ultimate stiffness is provisionally taken as a percentage of the initial compressive stiffness $(-25 \%$ in this study).

Under tension, if no unloading has previously taken place in tension, the pre-ultimate regime of the tensile LSC follows the path 'permanent deformation point $\rightarrow$ tensile ultimate point'. If any unloading has previously taken place in tension, the pre-ultimate regime of the tensile LSC follows the path 'permanent deformation point $\rightarrow$ tensile unloading point $\rightarrow$ tensile ultimate point'. In the post-ultimate regime, the stiffness is taken as zero.

\subsubsection{Updating rule}

Once the unloading is detected on an element, the last applied strain and resultant stress are recorded as the unloading strain and stress respectively. Critical points of the LSC are updated in accordance with the following rules:

1. Update the permanent deformation point assuming that the unloading stiffness equals to the initial stiffness of the corresponding loading direction where the unloading takes place;

2. If there is no change on the permanent deformation point, the following update is not required and all of the critical points remain the same;

3 . If the unloading takes place beyond the ultimate point, the ultimate point is updated as the unloading point; if not, the ultimate point remains the same;

4. If the unloading takes place at compression, the critical points relevant to tension remain the same;

5. If the unloading takes place at tension, the critical points relevant to compression remain the same except for the compressive ultimate strain, which should be updated considering the change of the permanent deformation point.

\subsubsection{Schematic illustration}

The response rule and updating rule introduced above are schematically illustrated in Figure 4.

As shown in Figure 4(a), the unloading takes place in compression at Point $e$, which is beyond the compressive ultimate point (Point $d$ ). 


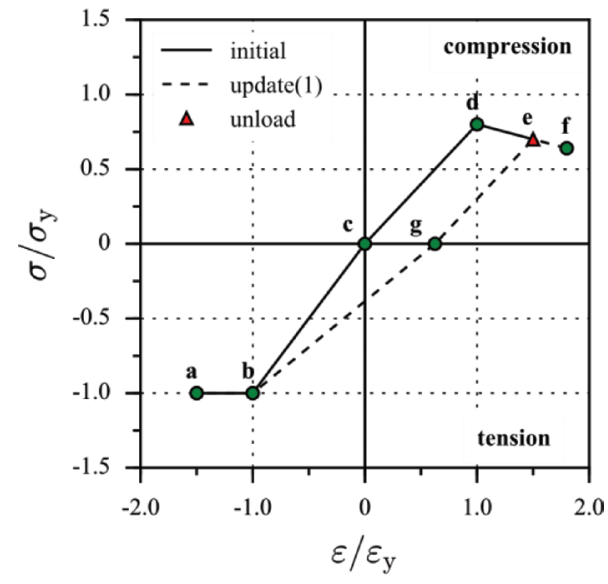

(a) LSC update 1

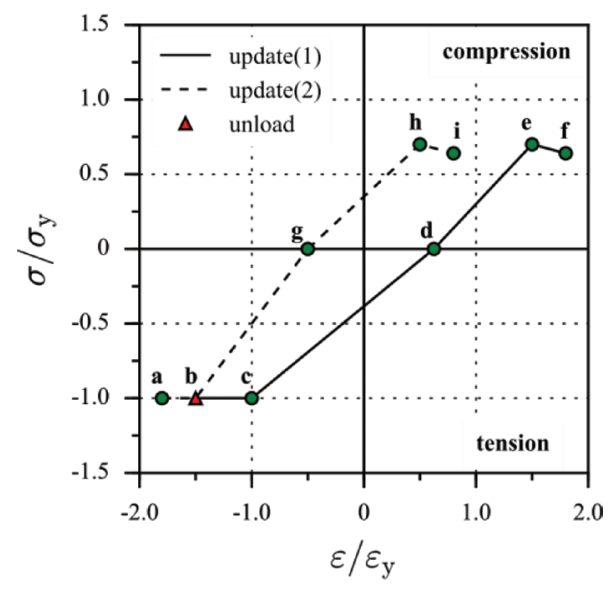

(b) LSC update 2

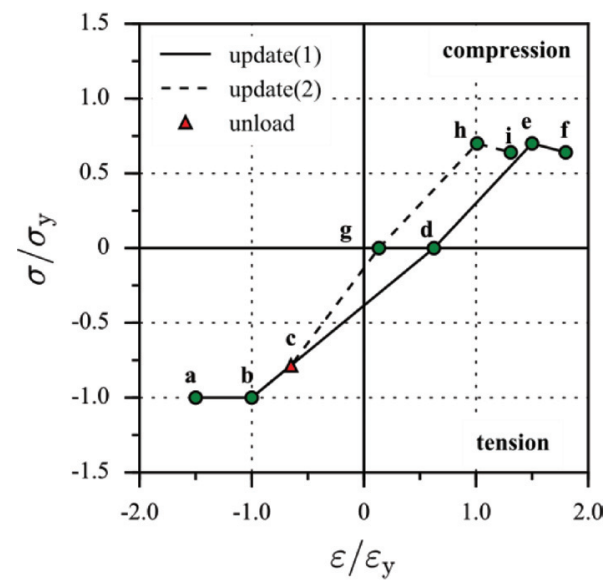

(c) LSC update 2*

Figure 4. Illustration of the updated LSC (Initial loading direction: compression).
The permanent deformation point is calculated as Point $g$ and the compressive ultimate point is updated as Point $e$ according to the updating rule $1 \& 3$ respectively. The tensile unloading point and ultimate point remain the same, as stated in the updating rule 4 . Hence, the LSC is re-formulated as $a \rightarrow b \rightarrow g \rightarrow e \rightarrow f$ in accordance with the response rule.

The next unloading, as shown in Figure 4(b), takes place in tension at Point $b$, which exceeds the tensile ultimate point. Similarly, the permanent point is predicted as Point $g$ and the tensile ultimate point is updated as Point $b$. In this case, the compressive unloading point and compressive ultimate strength remain the same. However, the compressive ultimate strain is shifted considering the change of the permanent deformation point according to the updating rule 5 .

Figure 4(c) illustrates a variation of the LSC update shown in Figure 4(b). In this case, the unloading in tension takes place before the tensile ultimate point. Therefore, the tensile ultimate point remains the same (Point $b$ ), following the updating rule 3 . This is also an illustration of why the response of tension is controlled by the permanent deformation point, unloading point and ultimate point. Otherwise, the tensile LSC in this case will be formulated as the path $b \rightarrow g$ instead of $b \rightarrow c \rightarrow g$. This is not an issue for compression, since the compressive ultimate point will be shifted in accordance with the change of permanent deformation point.

\section{CASE STUDY}

\subsection{Characteristics of the case study models}

Two box girder models are adopted for case study. Figures 5 and 6 show the cross sections of two models. Both models have a width of $12000 \mathrm{~mm}$,

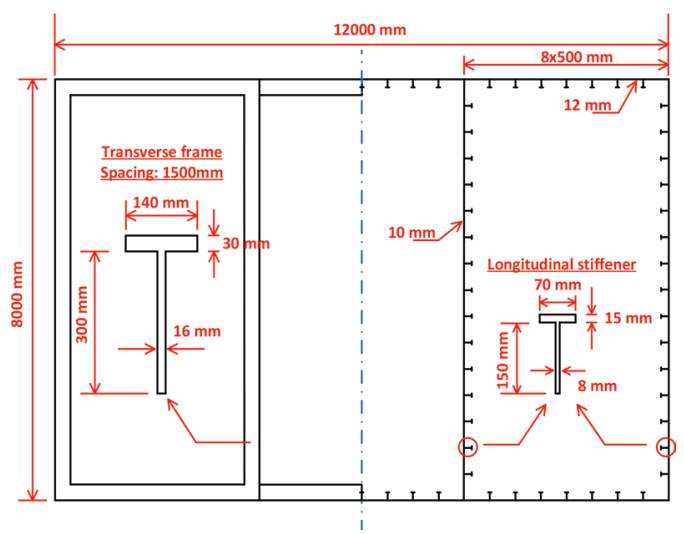

Figure 5. Single hull box girder cross section. 


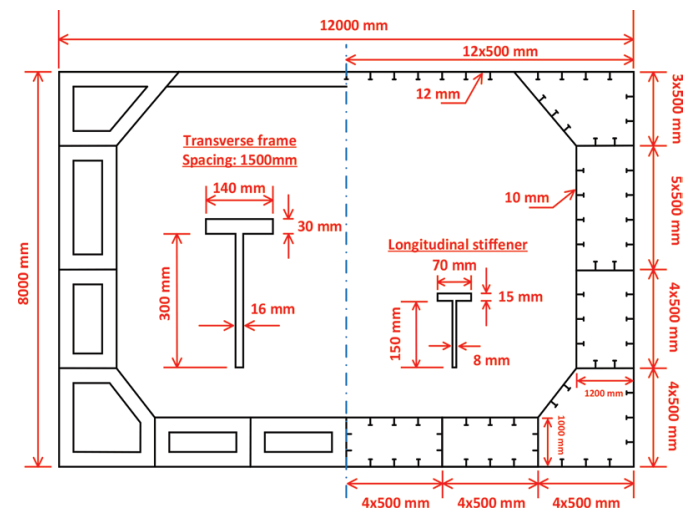

Figure 6. Double hull box girder cross section.

Table 1. Plate slenderness and column slenderness ratios of the stiffened panel elements.

\begin{tabular}{llll}
\hline & ID & $\beta$ & $\lambda$ \\
\hline Single hull & S1 & 1.63 & 0.31 \\
& S2 & 1.95 & 0.30 \\
Double hull & D1 & 1.63 & 0.31 \\
& D2 & 1.95 & 0.30 \\
& D3 & 1.55 & 0.29 \\
& D4 & 1.67 & 0.29 \\
& D5 & 1.56 & 0.29 \\
& D6 & 1.30 & 0.68 \\
\hline
\end{tabular}

Table 2. Loading protocols.

\begin{tabular}{ll}
\hline & Applied curvature $(\mathrm{rad} / \mathrm{mm})$ \\
\cline { 2 - 2 } Case & $0 \rightarrow \phi_{\text {sagging }} \rightarrow 0 \rightarrow \phi_{\text {hogging }}$ \\
\hline 1 & $6 \times 10^{-7} \rightarrow-6 \times 10^{-7}$ \\
2 & $6 \times 10^{-7} \rightarrow-3 \times 10^{-7}$ \\
3 & $3 \times 10^{-7} \rightarrow-6 \times 10^{-7}$ \\
\hline
\end{tabular}

a height of $8000 \mathrm{~mm}$ and are transversely framed every $1500 \mathrm{~mm}$. The thicknesses of the outer and the inner plating are $12 \mathrm{~mm}$ and $10 \mathrm{~mm}$ respectively.

The material yield stress is $315 \mathrm{MPa}$ and Young's modulus is $207000 \mathrm{MPa}$. An elastic-perfectly plastic behaviour is assumed. The plate and column slenderness ratios of the stiffened panel elements are listed in Table 1. Hard corners are introduced as the nearest plate element with respect to each intersection.

Three different loading protocols are analyzed for each model, given in Table 2 in terms of the applied curvature range. In case 1, the box girder is equally loaded in sagging and hogging. In case 2 , a varied amplitude loading protocol is applied where the unloading in hogging is started before the ultimate point. In case 3, the box girder is first loaded in sagging near the ultimate point, followed by a hogging beyond the ultimate limit state.

\subsection{Result and discussion}

The bending moment-curvature responses predicted by the proposed methodology as well as the NLFEA validation are plotted from Figures 7 to 12.

The NLFEA is performed on a $1 / 2+1+1 / 2$ bay model with a characteristic mesh size of $50 \mathrm{~mm}$ $\times 50 \mathrm{~mm}$ using the dynamic explicit solver. However, the analysis for double hull (Case 3 ) adopts a $100 \mathrm{~mm} \times 100 \mathrm{~mm}$ element size to avoid the instability in finite element analysis. A mesh conver-

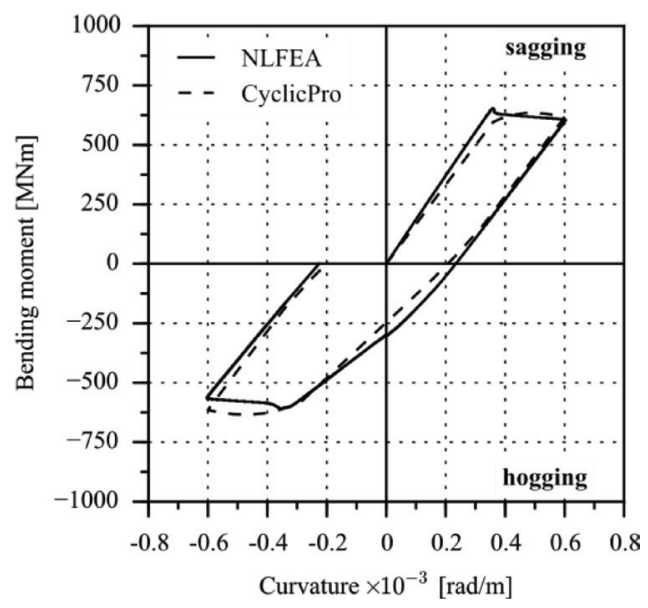

Figure 7. M-C curve (single hull model, Case 1).

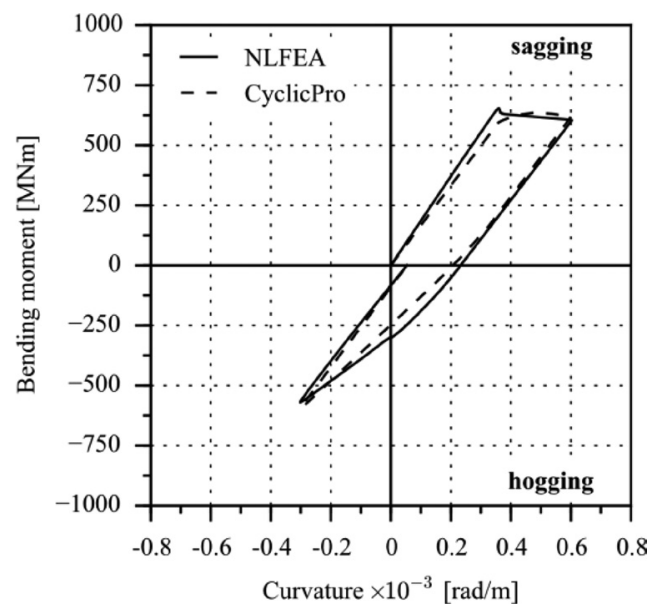

Figure 8. M-C curve (single hull model, Case 2). 


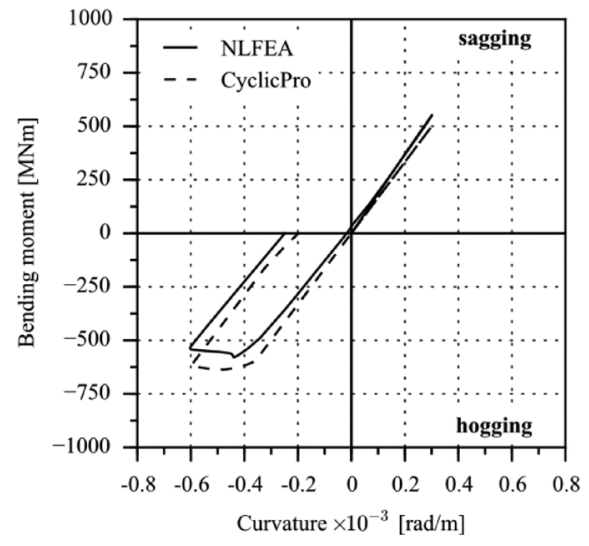

Figure 9. M-C curve (single hull model, Case 3).

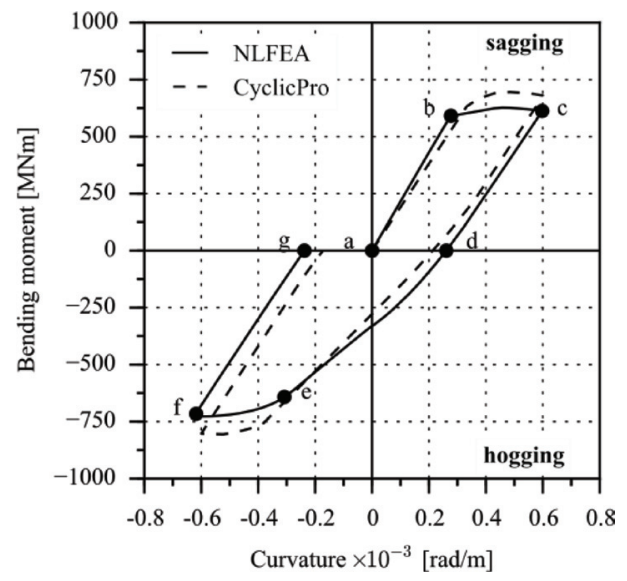

Figure 10. M-C curve (double hull model, Case 1).

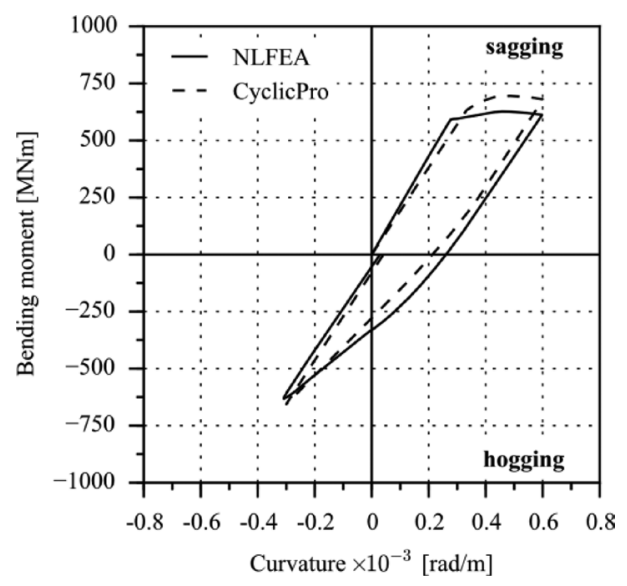

Figure 11. M-C curve (double hull model, Case 2).

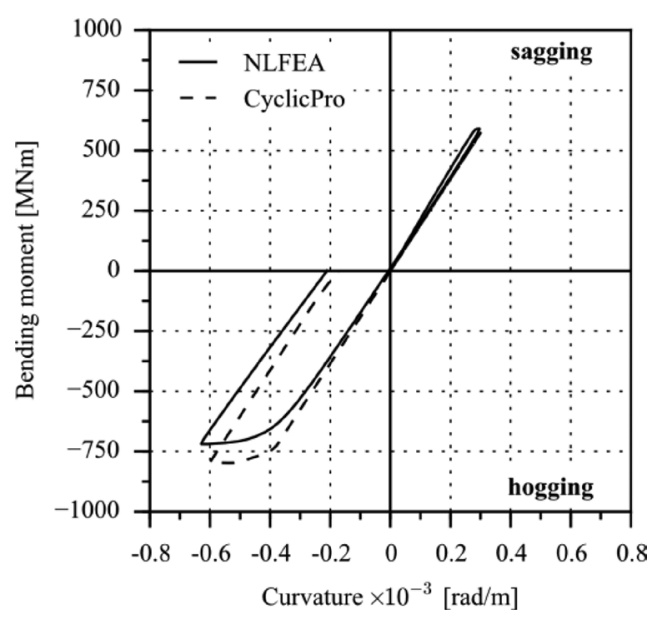

Figure 12. M-C curve (double hull model, Case 3).

gence study has shown that this change on mesh size will not drastically affect the analysis result.

To ensure that the buckling nucleates at the central bay, the initial geometric imperfection is only introduced on the central bay, including a local plate deflection, stiffener sideway deflection and column-type deflection. Welding-induced residual stress is not considered. The rotational control method with a reference point arbitrarily created at one end is used for the loading application, while the opposite end is fixed in six degrees of freedom.

In general, a close agreement is shown on the results predicted by two methods in terms of the initial loading, unloading and reloading responses. Additionally, the response predicted either by the proposed cyclic progressive collapse method or the NLFEA indicates that it is not justifiable to simplify the reloading bending response as the same as the initial path, which is assumed in the hydro-elasoplasticity method (Iijima et al., 2011). As shown in Figure 13 where the average stiffness of each path is compared for the result of double hull under case 1 loading protocol (Figure 11), the stiffness of the reloading path $(\mathrm{d} \rightarrow \mathrm{e})$ is only a half of the initial stiffness $(a \rightarrow b)$.

Typical load-shortening curve of structural element and NLFEA validation using a plate-stiffener combination model are compared in Figure 14. It should be recalled that, unlike the monotonic progressive collapse analysis, the exact loading protocol of each element in the cyclic progressive collapse method is unknown in advance. This comparison can only be carried out after the hull girder response prediction.

The simplified LSC curve has a noticeably lower initial stiffness. This is due to the fact that the adopted empirical formula is relatively con- 


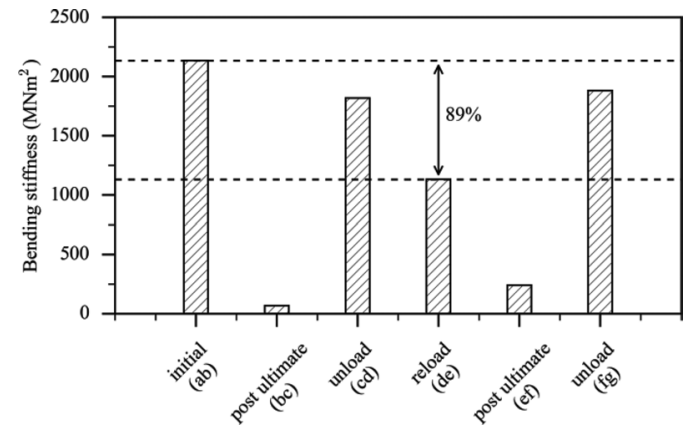

Figure 13. Example of the variation of the bending stiffness (Double hull, case 1, NLFEA).

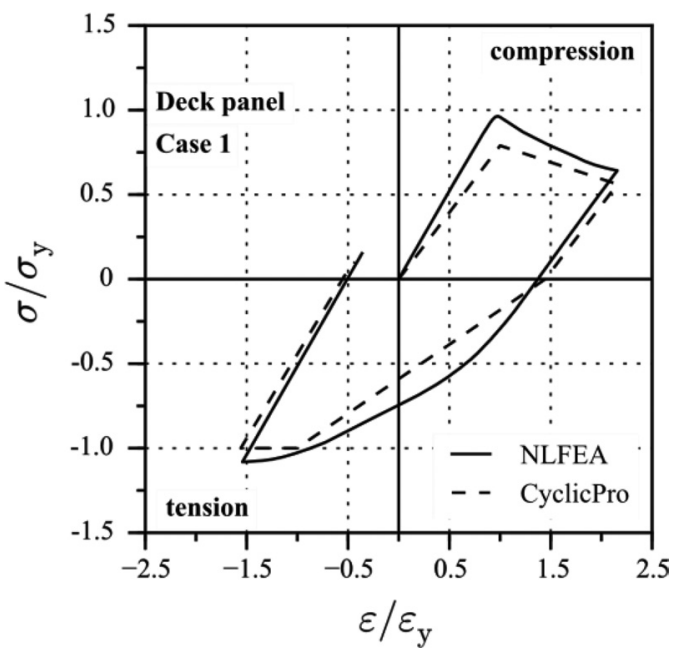

Figure 14. LSC comparison (Double hull, case 1).

servative and the pre-ultimate response is assumed as linear. This underestimation of initial stiffness leads to a lower initial bending stiffness of the hull girder.

On the other hand, the transition between compression and tension is not well predicted. As shown in Figure 14, the FEA predicts that the element retains the same stiffness at this compression/ tension conjunction and smoothly transit to the tensile ultimate point, whereas the tensile stiffness of the simplified curve is much lower. This LSC difference results in an underestimation of the hull girder collapse extent (permanent curvature).

\section{RECOMMENDATION}

Two challenges have been identified in the case study, namely the underestimation of the initial stiffness and the compression/tension transition.
Further, the post-ultimate stiffness of compression is taken as $-25 \%$ of the initial stiffness, which has a close agreement with the FEA prediction in the present case study. But it will be more sensible to relate the post-ultimate stiffness with the geometric parameters. In addition, the multi-cycle behaviour needs to be further investigated. This proposed method assumes that the subsequent compressive ultimate strength will always reduce to the compressive unloading stress, which means there will be a large strength reduction accumulated after several cycles of loading. In fact, a stabilization phenomenon of the compressive response occurs after two or three cycles, as indicated by Yao and Nikolov (1990). Besides, FEA shows that tensile ultimate point slightly deviate from the tensile unloading point during the load cycle. But the present study assumes they are always overlapped.

In this regard, it is recommended that the IACS method, nonlinear finite element method and other analytical method should be used to replace the simple compressive bilinear LSC. Additionally, a further parametric investigation on the cyclic behaviour of structural component is required to clarify the compressive response stabilization, strength and stiffness variation and compression/ tension transition.

\section{CONCLUSIONS}

A cyclic progressive collapse method is proposed to predict the bending response of a ship hull girder. A validation is carried out with equivalent finite element analysis. The following conclusions are drawn from this study:

- It is feasible to extend the simplified progressive collapse method to predict the cyclic bending response of a ship hull girder through the reformulation of LSC.

- The simple empirical formula-based LSC in conjunction with the present re-formulation scheme is suitable for the prediction of singlecycle bending response.

- The unloading bending stiffness is slightly lower than the initial bending stiffness, but the reloading bending stiffness is considerably lower than the initial bending stiffness

- Further study is required on the multi-cycle behaviour of structural component.

\section{REFERENCES}

14th ISSC, 2000. Ultimate Strength. ISSC Committee III.1. Nagasaki, Japan.

15th ISSC, 2003. Ultimate Strength. ISSC Committee III.1. San Diego, United States. 
20th ISSC, 2018. Ultimate Strength. ISSC Committee III.1. Delft, Netherlands.

Caldwell, J.B., 1965. Ultimate longitudinal strength. Trans., RINA, 107, 411-430.

Cui, H., Yang, P., 2018. Ultimate strength and failure characteristics research on steel box girders under cyclic-bending moments. Journal of Marine Science and Technology.

Derbanne, Q., Lauzon, J., de, Bigot, F., Malenica, S., 2016. Investigations of the dynamic ultimate strength of a ship's hull girder during whipping. In Proceedings: International Symposium on Practical Design of Ships and others Floating Structures (PRADS), Copenhagen, Denmark.

Dow, R.S., 1991. Testing and analysis of a 1/3 scale frigate model, in advances in marine structures 2. Dunfermline, Scotland: Elsevier, 749-773.

Fukumoto, Y., Kusama, H., 1985. Cyclic bending tests of thin-walled box beams. In Proceedings of JSCE, Structural engineering/Earthquake engineering, 2.

Iijima, K., Kimura, K., Xu, W., Fujikubo, M., 2011. Hydroelastoplasticity approach to predicting the post-ultimate strength behaviour of a ship's hull girder in waves. Journal of Marine Science Technology, 16(4): 379-389.

Iijima, K., Fujikubo, M., 2015. Cumulative collapse of a ship hull girder under a series of extreme wave loads. Journal of Marine Science and Technology, 20 (3): 530-541.

Kaminski, M., L., 1992. Cyclic compression of imperfect plates. PhD thesis, Delft University of Technology.
Paik, J.K., Thayamballi, A.K., 1997. In Proceedings: An empirical formulation for predicting the ultimate compressive strength of stiffened panels. International Conference on Offshore and Polar Engineering, 328-338 Honolulu, Hawaii.

Hess, P.E., Adamchak, J.C., Falls, J., 1997. Failure analysis of an inland waterway oil bunker tanker. Survivability, structures, and material directorate technical report. Naval surface warfare center, Carderock division.

Smith, C.S., 1977. Influence of local compressive failure on ultimate longitudinal strength of a ship's hull. In Proceedings, International Symposium on Practical Design of Ships and others Floating Structures (PRADS), Tokyo, Japan.

Sumi, Y., Fujikubo, M., Fujita,H., Kawagoe, Y., Kidogawa, M., Kobayashi, K., Nakano, T., Iwano, J., Takahira, T., Tamura, K., Ueda, N., 2015. Final Report of Committee on Large Container Ship Safety, Japan.

Yao, T., Nikolov, P., I., 1990. Buckling/plastic collapse of plates under cyclic loading. Journal of the Society of Naval Architects of Japan, 168.

Yao, T., Fujikubo, M., Nie, C., Kamiyama, S., 1995. Development and application of simple plate model to simulate collapse behaviour under thrust. Journal of the Society of Naval Architects of Japan, 178, 439-449 (in Japanese). 